\title{
Saudi experts' recommendation for RSV prophylaxis in the era of COVID-19
}

\author{
Consensus from the Saudi Pediatric Pulmonology Association
}

Adel S. Alharbi, MD, FCCP, Mohamed Alzahrani, MBBs, MD, Abdulrahman N. Alodayani, MBBS, MD,

Mohamed Y. Alhindi, MBBS, MD, Saleh Alharbi, MBBS, MD, Abdulrahman Alnemri, MBBS, MD.

\begin{abstract}

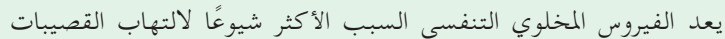

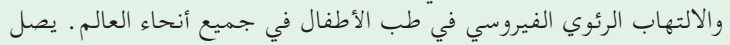

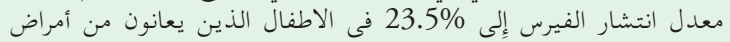

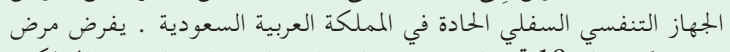

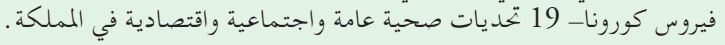

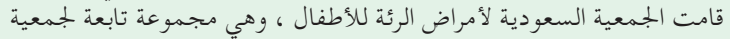

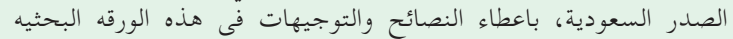

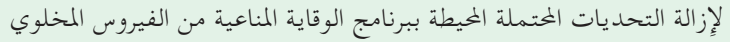

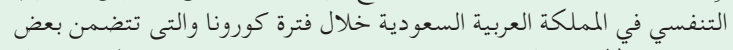

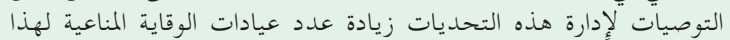

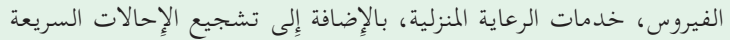

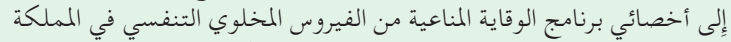

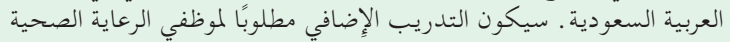

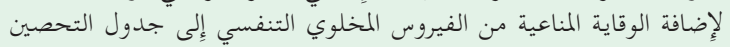

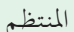

Respiratory syncytial virus (RSV) is the most common cause of bronchiolitis and viral pneumonia in pediatrics worldwide. In the Kingdom of Saudi Arabia (KSA), the prevalence of RSV is $23.5 \%$ in pediatric patients with acute lower respiratory tract illness. Coronavirus disease (COVID-19) poses critical public health and socioeconomic challenges in KSA. The Saudi Pediatric Pulmonology Association (SPPA), a subsidiary of the Saudi Thoracic Society (STS), developed a task force to determine the potential challenges and barriers to the RSV immunoprophylaxis program during the era of COVID-19 and to compose a practical, nationwide, and multidisciplinary approach to address these challenges. Some of the recommendations to manage these challenges include increasing the number of RSV immunoprophylaxis clinics, drive-thru visits, home-care services, and swift referrals to the RSV immunoprophylaxis program specialists. Additional training is required for healthcare personnel to add RSV immunoprophylaxis to the regular immunization schedule.

Keywords: respiratory syncytial virus, immunoprophylaxis, RSV, COVID-19
Saudi Med J 2021; Vol. 42 (4): 355-362

doi: 10.15537/smj.2021.42.4.20200769

From the Department of Pediatrics (Alharbi A, Alodayani), Prince Sultan Military Medical City; from the Department of Pediatrics (Alzahrani), Security Forces Hospital; from the Pediatrics Department (Alnemri), College of Medicine, King Saud University, Riyadh; from the Department of Pediatrics (Alhindi), King Abdullah International Medical Research Center, King Saud Bin Abdulaziz University for Health Sciences, Ministry of National Guard; from the Department of Pediatrics (Alharbi S), Dr. Soliman Fakeeh Hospital, Jeddah; and from the Department of Pediatrics (Alharbi S), Umm Al-Qura University, Mecca, Kingdom of Saudi Arabia.

Received 28th November 2020. Accepted 23rd February 2021.

Address correspondence and reprint request to: Dr. Adel S. Alharbi, Department of Pediatrics, Prince Sultan Military Medical City, Riyadh, Kingdom of Saudi Arabia. E-mail: dr.adelalharbi@gmail.com ORCID ID: https://orcid.org/0000-0001-7974-4026

1. Introduction. Respiratory syncytial virus (RSV) is the most common cause of bronchiolitis and viral pneumonia in pediatric patients and is the leading cause of lower respiratory tract illness in infants' first year of life. ${ }^{1,2}$ Approximately, $70 \%$ of bronchiolitis cases stem from an RSV infection. ${ }^{3}$ According to the World Health Organization (WHO), the estimated annual burden of RSV-related acute lower respiratory illness (ALRI) is approximately 33 million worldwide, with approximately 3 million hospitalizations and 59,600 deaths. ${ }^{4}$ Respiratory syncytial virus infection represents a grave condition in developing countries, with approximately 99\% of RSV-related deaths and $93 \%$ of RSV-related ALRI deaths occurring in developing countries. ${ }^{5}$ The prevalence of RSV infection is high in the Middle East and North Africa (MENA). In a systematic review of all RSV-related articles in the MENA region from 2001 to 2019, high annual incidence rates of RSV were reported in Jordan (64\%), followed by Pakistan (52.6\%), Tunisia (50\%), Qatar 
(48.5\%), Algeria (47.8\%), Egypt (46.6\%), and Iran (46.1\%), whereas the lowest incidence was reported in Oman (1.8\%). ${ }^{2}$ In the Kingdom of Saudi Arabia (KSA), the prevalence of RSV infection is $23.5 \%$ in pediatric patients with ALRI. ${ }^{6}$

The RSV is highly contagious, with a $98 \%$ risk (attack rate) in cases of first-time infection during outbreaks and $75 \%$ in cases of reinfection. ${ }^{7}$ Transmission usually occurs through close contact or exposure of the nasal mucosa or conjunctiva to the patient's droplets or secretions. This knowledge can be useful for limiting the spread of the virus, where isolation and quarantine measures can be used to reduce the virus transmission. ${ }^{8}$

The pathophysiology of bronchiolitis is characterized by heightened mucus production, widespread inflammation, edema of the airways, and necrosis of the respiratory epithelial cells. ${ }^{9}$ The destruction of airway epithelial cells is caused by the RSV binding to the epithelial cells and subsequently replicating, causing necrosis and ciliary destruction. ${ }^{10}$ This cell destruction triggers an immune response characterized by the proliferation of lymphocytes and polymorphonuclear cells (PMNCs), while increased mucus secretion results in the generation of edematous submucosa and adventitial tissues. The cascade of these events leads to bronchiolar obstruction, air trapping, and various degrees of lobar collapse. ${ }^{10}$

Management strategies for viral bronchiolitis vary widely between different studies; however, there has been a recent shift in favor of minimal supportive therapy. ${ }^{3,11}$ This type of minimally invasive intervention involves nasopharyngeal suction, oxygen therapy, and sustained hydration with fluid management. Other forms of respiratory support include high-flow nasal cannula (HFNC) oxygen, continuous positive airway pressure (CPAP), mechanical ventilation, heliox, and chest physiotherapy. Evidence of the efficacy of pharmacological agents in treating bronchiolitis is generally scarce; ${ }^{3}$ however, immunoprophylaxis using the monoclonal antibody palivizumab is the recommended route for RSV infection prevention.

Disclosure. Authors have no conflict of interests, and the work was not supported or funded by any drug company. Dr. Adel S. Alharbi is a member of the Editorial Team, and was therefore excluded from any final editorial decisions regarding this paper.
Additionally, limited handling is preferred to avoid infant exhaustion, especially because infants are generally intolerant of interventions. ${ }^{3}$

Premature infants born prior to 37 weeks of gestational age (wGA) are particularly prone to severe morbidities due to anatomical, metabolic, and immunological predisposing characteristics. ${ }^{12}$ As mentioned previously, RSV infection is the leading cause of hospitalizations for lower respiratory tract infections (LRTI) in infants. It is imperative that premature infants receive adequate prophylactic care to avoid severe bronchiolitis. ${ }^{12}$ The Food and Drug Administration (FDA) approved palivizumab in 1998, a monoclonal antibody that remains, to date, the main prophylactic intervention against RSV infection. ${ }^{13}$ Since its inaugural licensure, the American Academy of Pediatrics (AAP) has updated its RSV immunoprophylaxis guidelines 5 times, most recently in 2014. Current AAP guidelines limit RSV immunoprophylaxis to premature infants born at $<29$ wGA, infants $<32$ wGA with chronic lung disease (CLD), and infants $<12$ months with chronic heart disease (CHD). ${ }^{13}$ Up to 5 monthly doses should be administered over the course of the RSV season, ${ }^{14}$ which could be a challenge in areas with active COVID-19 lockdown and strained healthcare services. The AAP 2014 guideline was based on the cost-effectiveness ratios for RSV immunoprophylaxis. ${ }^{15}$

The AAP 2014 guideline recommends that premature infants who previously qualified to receive immunoprophylaxis under the 2012 guidelines do not receive immunoprophylaxis (without other qualifying conditions). Besides, infants with CHD aged less than one year are no longer recommended to get RSV immunoprophylaxis according to the AAP 2014 guideline, while the 2012 guidelines recommended the RSV immunoprophylaxis for infants with CHD aged $<2$ years at the beginning of RSV season. ${ }^{16}$

2. Respiratory syncytial virus epidemiology, immunoprophylaxis, and management in KSA. The number of children $<5$ years of age who succumb to RSV-associated ALRI ranges from 66,000 to $1,99,000$, with $99 \%$ of the deaths occurring in developing countries. Respiratory syncytial virus is endemic to some areas, particularly South American countries. Data from the southern hemisphere show that RSV immunity is effective against other viruses as well (such as, Argentina). High-risk patients for RSV include premature infants and infants with chronic lung diseases or cardiac abnormalities. Respiratory syncytial virus-related LRTI in early life is a significant risk factor for developing persistent wheezing and asthma through 
early childhood. The monoclonal antibody palivizumab confers passive immunity against RSV.

There are more than 5 million (15.4\%) LRTI cases in the KSA. ${ }^{17}$ In 1991-1992, respiratory viruses were identified in approximately $62 \%$ of the 127 children with ALRI in Riyadh, and of these, 54\% were positive for RSV. ${ }^{18}$ In a comprehensive study by Al-Hajjar et al ${ }^{19}$ carried out in Riyadh, KSA a total of 1429 samples were tested for respiratory viruses, and the RSV was the most common viral pathogen detected (29\%). In a study by Al-Qassim, RSV was detected in 45\% of 282 children with ALRI during 2003-2004. ${ }^{20}$

3. Status of RSV immunoprophylaxis program. Patients requiring the injection have easy access to the immunization program in the KSA. The new pre-mixed formulation of the RSV immunoprophylaxis injection is liquid and ready to use. Additionally, pharmacists need to relinquish the exclusive privilege of preparing the injection and allow clinics to do it as well to ensure maximum availability. In terms of availability, insurance companies did not entirely cover high-risk groups in the past. This was rectified when the guidelines came out, as insurance companies started accepting the vast majority of palivizumab requests. This was further facilitated by the drastic reduction in time, resulting from the new ready-to-administer liquid formulation.

There was a significant improvement in the national RSV immunoprophylaxis program after the Saudi initiative of bronchiolitis diagnosis, management, and prevention (SIBRO) program was rolled out in July 2018. The Saudi Council of Cooperative Health Insurance revised its policy to include the RSV immunoprophylaxis program as a mandatory requirement in the Saudi healthcare system.

4. Saudi Initiative of Bronchiolitis Diagnosis, Management, and Prevention (SIBRO). In July 2018, the SIBRO guidelines were declared by the Saudi Pediatric Pulmonology Association (SPPA) - a subdivision of the Saudi Thoracic Society (STS) - when they found that RSV infection was widespread in the country and the national guidelines according to the most recent and the best evidence-based practice was needed urgently. ${ }^{11}$

The SIBRO developed evidence-based guidelines for using palivizumab immunoprophylaxis in different patient categories (Tables $1 \& 2$ ). The SIBRO team focused mainly on high-risk groups such as premature infants and children with CLD and cardiac abnormalities. It was a great achievement for the SPPA to release such an important national guideline because the adoption of external guidelines might carry some concern as the management of RSV infection is always a mix of actions based on the published evidence and the local practice.

5. Respiratory syncytial virus immunoprophylaxis programs in the era of COVID-19 in the KSA. 5.1. Burden of COVID-19 in the KSA. The coronavirus pandemic is the most recent outbreak caused by the severe acute respiratory syndrome coronavirus-2 (SARSCoV-2) in Wuhan, China. ${ }^{21}$ The infection caused by the SARS-CoV-2 is called "coronavirus disease-19" (COVID-19). Other versions of coronaviruses have emerged at various points, such as those responsible for the SARS outbreak in 2002 and Middle East respiratory syndrome (MERS) outbreak in 2012. As of November 21, 2020, the SARS-CoV-2 reached 219 countries, infecting over $55,961,380$ persons, resulting in over 1,343,700 deaths worldwide. ${ }^{22}$ The devastating impact of the virus has been far-reaching, causing a significant strain on healthcare systems worldwide and major economic losses. ${ }^{23,24}$ The draining of healthcare resources by COVID-19 impacts the quality and availability of healthcare for other conditions, including RSV diagnosis, management, and immunoprophylaxis.

The KSA is one of the most severely affected countries with COVID-19 in the MENA region. As of September 21,2020 , the total disease burden reached over 330,246 cases, with over 4,512 deaths. ${ }^{22}$ The first confirmed case in the KSA was reported on March 2, 2020, but decisive social distancing measures were applied even before the first case was reported. These measures ranged from suspension or cancellation of sporting, religious, and entertainment gatherings to temporary closure of mass gathering at the Umrah, mosques, and educational establishments and even total curfew. ${ }^{25}$ The lockdown measures implemented by the Saudi government have been largely successful in keeping infection levels under control. A study published in April 2020 predicted that the number of infections in the KSA could be limited to 0.4 million cases by September $2020 .{ }^{26}$ The current number of cases is still below 0.4 million, which is a sign of effective management of the local virus transmission

5.2. COVID-19 infection in the pediatric population in KSA. Children of all ages can contract COVID-19. However, children younger than 12-14 years appear to be affected less frequently than adults. In surveillance data from various countries, children typically account for $1-9 \%$ of laboratory-confirmed cases. In the KSA, children account for almost $8 \%$ of all confirmed COVID-19 cases; the increased rate was reported from the beginning of the pandemic; according to statistics 
Table 1 - Saudi Initiative of Bronchiolitis Diagnosis, Management, and Prevention recommendations on using Palivizumab across different patients' categories. ${ }^{11}$

\begin{tabular}{|c|c|c|}
\hline Patient segment & Recommendations & Level of evidence \\
\hline Early preterm (= 28 weeks, 6 days GA) & $\leq 12$ months of age & 1B \\
\hline $\begin{array}{l}\text { Mid preterm ( } 29 \text { weeks GA, } 0 \text { days to } 32 \\
\text { weeks, } 6 \text { days GA) }\end{array}$ & $\leq 6$ months of age & $1 \mathrm{~B}$ \\
\hline $\begin{array}{l}\text { Late preterm ( } 33 \text { weeks, } 0 \text { days weeks GA } \\
\text { to } 35 \text { weeks, } 0 \text { days GA) }\end{array}$ & $\begin{array}{l}\leq 6 \text { months of age at the start of the RSV season OR born during RSV season with } \\
\text { at least one of the following risk factors: } \\
\text { Attendance at child care } \\
\text { Children }<5 \text { years of age who live permanently in the same household (including } \\
\text { siblings) } \\
\text { Exposure to environmental air pollutants }\end{array}$ & $1 \mathrm{~B}$ \\
\hline Infants and children with CLD & $\begin{array}{l}<12 \text { months for all; }<24 \text { months if still receiving medications for CLD within } 6 \\
\text { months from the beginning of the epidemic season }\end{array}$ & $1 \mathrm{~B}$ \\
\hline $\begin{array}{l}\text { Infants and children with } \\
\text { hemodynamically significant } \mathrm{CHD}\end{array}$ & $\begin{array}{l}<12 \text { months for all; }<24 \text { months if still receiving medications for the cardiac } \\
\text { condition }<6 \text { months from the beginning of the epidemic season. } \\
\text { Postoperative dose after cardio bypass }\end{array}$ & $1 \mathrm{~B}$ \\
\hline $\begin{array}{l}\text { Children with anatomic pulmonary } \\
\text { abnormalities or neuromuscular disorders }\end{array}$ & $\begin{array}{l}<24 \text { months may be considered for infants with impaired ability to handle } \\
\text { respiratory secretions. }\end{array}$ & $3 \mathrm{~B}$ \\
\hline Immunocompromised children & $\begin{array}{l}<24 \text { months may be considered for children who are profoundly } \\
\text { immunocompromised during the RSV season }\end{array}$ & $2 \mathrm{~B}$ \\
\hline Children with down syndrome & $\begin{array}{l}\text { Recommended for children with accompanying qualifying heart disease, CLD, } \\
\text { airway clearance issues, or premature birth ( }<35 \text { weeks, } 0 \text { days GA) }\end{array}$ & $2 \mathrm{~B}$ \\
\hline Children with cystic fibrosis & $\begin{array}{l}<12 \text { months with clinical evidence of CLD and/or nutritional compromise for } \\
<24 \text { months with manifestations of severe lung disease or } \\
\text { weight for length }<10^{\text {th }} \text { percentile }\end{array}$ & $2 \mathrm{~A}$ \\
\hline $\begin{array}{l}\text { Special situations: If an infant who } \\
\text { is receiving prophylaxis experiences a } \\
\text { breakthrough of RSV }\end{array}$ & $\begin{array}{l}\text { If an infant who is receiving prophylaxis experiences a breakthrough of RSV, the } \\
\text { monthly prophylaxis should continue as planned until a maximum of } 5 \text { doses } \\
\text { have been administered }\end{array}$ & $3 \mathrm{~B}$ \\
\hline
\end{tabular}

published by the Ministry of Health $(\mathrm{MOH})$, the rate of children with COVID-19 increased from $4.8 \%$ in March 2020 to $12 \%$ at the end of May. 27,28 In Jeddah, in a retrospective study conducted from March 1 to May 13, 2020, involving 191 patients aged below 18 years screened for COVID-19 by RT-PCR, 24 results were positive. The patients' ages ranged from 13 days to 17 years (average, 8.4 years; standard deviation, 5.87 years)..$^{29}$ Most cases of infection in children were attributable to household exposure, usually with an adult as the index patient. Infected children shed SARS-CoV-2 virus with nasopharyngeal viral loads comparable to or higher than those shed by adults. ${ }^{30}$

In the pediatric population, neonates are vulnerable to COVID-19 infection; frequently, they are infected by respiratory droplets or secretions during the postnatal period when they exposed to their mothers or other caregivers infected with the SARS-CoV-2. Approximately, $2-5 \%$ of the infants born to mothers with positive COVID-19 status show positive results on SARS-CoV-2 testing in the first 24-96 hours of life. To reduce the risk of transmission from mother to newborn, the $\mathrm{MOH}$ neonatology services improvement program published guidelines for neonates born to mothers with suspected or confirmed COVID-19 infection. ${ }^{31}$ Furthermore, a recent study from a single tertiary care center in Riyadh, KSA showed the immense impact of the COVID-19 pandemic on childhood vaccinations, mainly the significant drop in vaccination visits during April and May 2020. From March to May 2020, there were drops in the vaccination visits of $49.93 \%, 71.90 \%$, and $68.48 \%$, respectively, compared to the same period from 2017 to $2019 .^{32}$

\subsection{Consensus development for RSV} immunoprophylaxis program in KSA in the era of COVID-19. The Saudi Pediatric Pulmonology Association (SPPA) developed a taskforce to determine the potential challenges and barriers to the RSV immunoprophylaxis program in the KSA during the era of COVID-19 and to compose a practical, nationwide, multidisciplinary approach to address these problems. This initiative involved multi-multidisciplinary teams of pediatric pulmonologists, pediatric infectious disease specialists, and neonatologists from both the 
Table 2 - Respiratory syncytial virus prophylaxis program. ${ }^{11}$

\section{Referral form}

Part A and B to be filled by referring physician and to be sent to Email: email of the coordinator Contact info of the coordinator (extension)

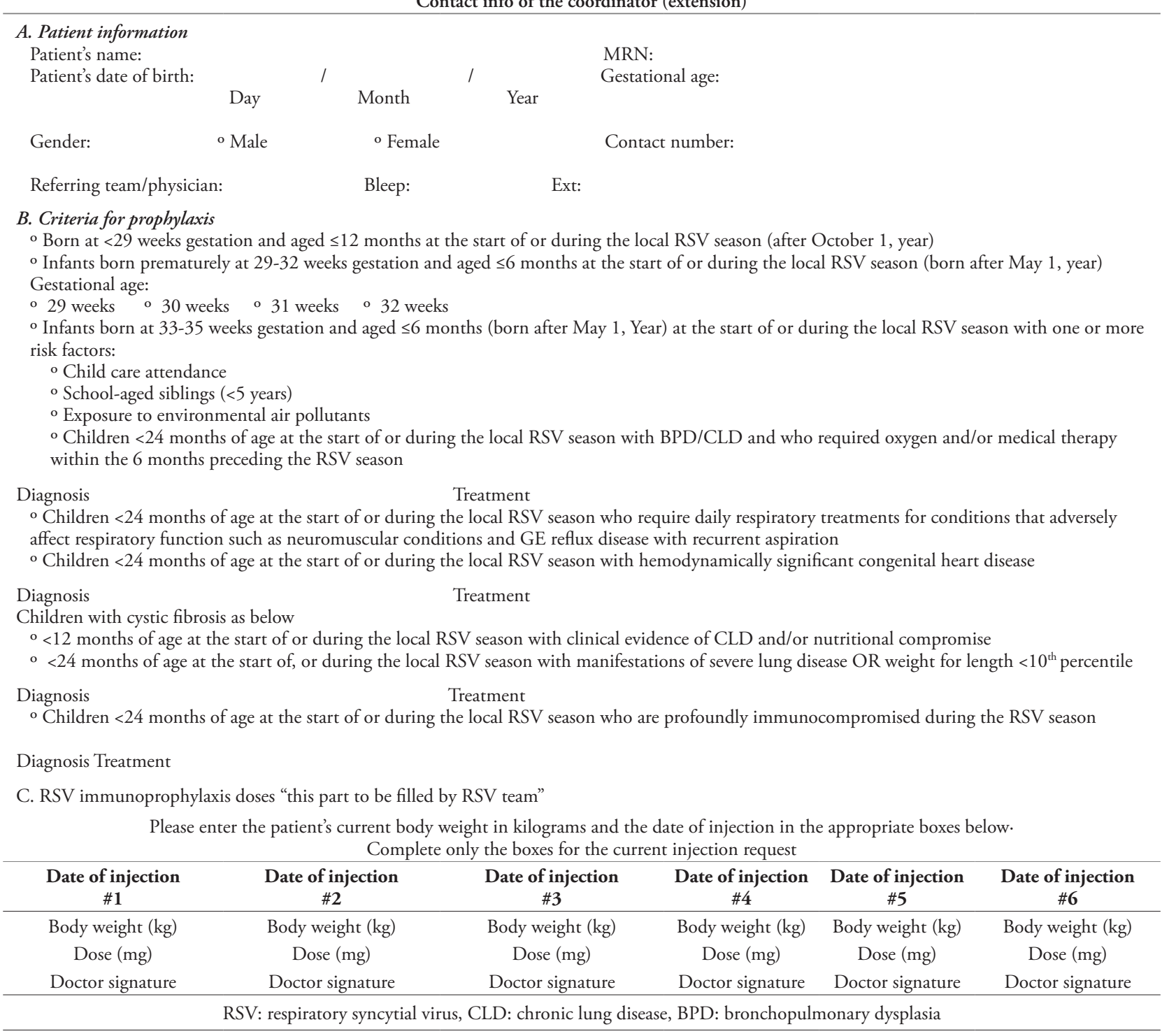

government and private sectors all over the KSA. Previously, the SIBRO also published a guideline on the diagnosis, management, and prevention of childhood bronchiolitis in the KSA. ${ }^{11}$

5.4. Respiratory syncytial virus immunoprophylaxis challenges in the era of COVID-19. Due to the current COVID-19 pandemic and the lockdown, infants and their parents are facing many difficulties in terms of visiting clinics. Additionally, there is a fear of going to the hospital due to the belief that hospitals are the main source of COVID-19, which creates compliance issues. Overloaded hospitals during a pandemic can expose infants to RSV, and this situation is complicated by the immune-deficient status of these high-risk infants. Another problem cause by the pandemic is that high-risk infants born a few months prior to the pandemic will be difficult to recall and might not receive the needed immunoprophylaxis. This situation is worsened by the sheer size of the KSA. 
5.5. Proposed solutions for RSV immunoprophylaxis program challenges:

I. Encourage referral sources (mainly neonatologists) to expedite the referral of high-risk patients to pediatric pulmonologists.

II. Dispense full course of palivizumab vials. However, this could cause some medication waste.

III. Discuss ways to improve home care quality with caregivers.

IV. Home vaccinations were successful during the pandemic at most of the major health care tertiary hospitals, reaching the number of vaccinations comparable to that reported last year (without COVID-19). Home vaccinations could be adopted for high-risk RSV infants to limit their exposure to the risk of RSV. However, this would require additional training of the vaccinating staff, especially in terms of limiting waste during injection preparation and administration.

V. Seek approval to open specialized RSV clinics in hospitals.

VI. Set up drive-thru clinics to facilitate injection administration.

VII. Minimize the number of patients per day and increase the number of clinics. This would require punctuality from the patients.

VIII. Set a specific appointment time for each patient and allow walk-in patients.

IX. Increase number of available days for clinic visits.

X. Include RSV immunoprophylaxis doses in the regular immunization schedule. This would require a separate clinic for RSV immunoprophylaxis programs to avoid exposing high-risk infants to RSV.

XI. Experts have seen success with this approach in their own hospitals, where extending the immunoprophylaxis clinic working hours help spread the appointments throughout the day and reduce crowding.

XII. Separate the RSV immunoprophylaxis program clinic from the regular vaccination areas.

XIII. Gather high-risk infants born during the pandemic as a group to administer their prophylactic injections.

6. Future of RSV amid COVID-19. Neonates are particularly susceptible to COVID-19, as $10 \%$ of coronavirus patients are pediatric patients, with $40 \%$ of them aged below 2 years. As such, experts predict challenging times ahead with the potential emergence of another COVID-19 wave, in addition to delays in the release of the seasonal flu vaccine. Conversely, the SIBRO's initiative to release clear RSV diagnosis and management guidelines has significantly reduced the number of infants who develop severe bronchiolitis, as per anecdotal accounts from experts. These guidelines will help address potential issues from another COVID-19 wave.

New promising treatment options for RSV infection have emerged in the recent years. Nirsevimab is an extended half-life monoclonal antibody that has been approved by FDA in February 2019 to be used for prevention of LRTI caused by RSV. ${ }^{33}$ It acts by the same mechanism of palivizumab through binding to the cells that express RSV protein. Nirsevimab is administered as a passive immunoprophylaxis agent to protect infants against RSV. Currently, the phase-3 trials are being conducted and anticipated to be published in 2023 (NCT03979313, NCT02878330). However, the phase-2 trials have shown a high efficacy in immunoprophylaxis against RSV. Infants who received Nirsevimab have shown a lower RSV LRTI and incidence of hospitalization compared to the infants who received placebo. ${ }^{34}$ Nirsevimab is preferable to be used over the current options as it only requires a single dose for the whole RSV season, unlike palivizumab who require a monthly injection. ${ }^{34}$ Also, Nirsevimab revealed a higher neutralizing activity and longer half-life than palivizumab.

The COVID-19 pandemic has highlighted the importance of epidemiological studies in the KSA. The KSA spared no expense to contain the pandemic and invested heavily into spreading awareness and enforcing precautionary protocols. This proactive approach can be applied to extremely low birth weight infants, where investment from the state can be a cost-effective measure to limit the spread of RSV. This investment can take the form of free RSV prophylaxis, which would help relieve the financial burden on the infant's family.

7. Conclusion. Respiratory syncytial virus mortality is an issue in developing countries, which can be exacerbated by the pandemic and overloaded healthcare institution. The closure or partial closure of clinics alongside a warranted fear of going to the hospital are the main challenges facing vulnerable patients. Some of the recommendations to manage these challenges include increasing the number of RSV immunoprophylaxis program clinics, drive-thru visits, and home vaccinations and encouraging swift referrals to specialists in the RSV immunoprophylaxis program. These solutions are not without faults, as additional training would be required for healthcare personnel to administer home 
vaccinations, and adding an RSV immunoprophylaxis program to the regular immunization schedule would exert an additional load on the healthcare system.

The new liquid form used in the RSV immunoprophy, which subsequently allowed for increased insurance coverage of high-risk infants. Including the RSV immunoprophylaxis doses into the regular immunization schedule can help mitigate RSV-related mortality; however, further examination of the benefits and feasibility of this recommendation is required. The short-term success of RSV immunoprophylaxis is threatened by the emergence of another COVID-19 wave, in addition to delays in the release of the seasonal flu vaccine. This presents an immense challenge to healthcare experts. State investments in RSV epidemiological studies and free vaccinations can help alleviate the brunt of the pandemic.

Acknowledgment. We would like to thank Saudi Thoracic Society, Saudi Arabia for their support. Also, we would like to thank Editage (www.editage.com) for the English language editing. Editorial support and medical writing of the manuscript was provided by Hussein Ahmed, MD and Ahmed Salah Hussein, MBBCH from RAY-contract Research Organization, Egypt.

\section{References}

1. Halasa N, Williams J, Faouri S, Shehabi A, Vermund SH, Wang L, et al. Natural history and epidemiology of respiratory syncytial virus infection in the Middle East: hospital surveillance for children under age two in Jordan. Vaccine 2015; 33: 6479-687.

2. Yassine HM, Sohail MU, Younes N, Nasrallah GK. Systematic review of the respiratory syncytial virus (RSV) prevalence, genotype distribution, and seasonality in children from the middle east and North Africa (MENA) region. Microorganisms 2020; 8: 713.

3. McNaughten B, Hart C, Shields M. Management of bronchiolitis in infants: key clinical questions. Paediatr. Child Health (United Kingdom) 2017; 27: 324-327.

4. World Health Organization. Who Strategy To Pilot Global Respiratory Syncytial Virus Surveillance Based On The Global Influenza Surveillance And Response System (GISRS). (Updated 2017; Cited). Available from URL: https://apps.who. int/iris/handle/10665/259853

5. Shi T, McAllister DA, O’Brien KL, Simoes EAF, Madhi SA, Gessner BD, et al. Global, regional, and national disease burden estimates of acute lower respiratory infections due to respiratory syncytial virus in young children in 2015: a systematic review and modelling study. Lancet 2017; 390: 946-958.

6. Albogami SS, Alotaibi MR, Alsahli SA, Masuadi E, Alshaalan M. Seasonal variations of respiratory viruses detected from children with respiratory tract infections in Riyadh, Saudi Arabia. J Infect Public Health 2018; 11: 183-186.

7. Henderson FW, Collier AM, Clyde WA, Denny FW. Respiratory-£ren. N Engl J Med 1979; 300: 530-534.

8. Wright M, Mullett CJ, Piedimonte G. Pharmacological management of acute bronchiolitis. Ther Clin Risk Manag 2008; 4: 895-903.
9. Florin TA, Plint AC, Zorc JJ. Viral bronchiolitis. Lancet 2017; 389: 211-224.

10. Aherne W, Bird T, Court SD, Gardner PS, McQuillin J. Pathological changes in virus infections of the lower respiratory tract in children. J Clin Pathol 1970; 23: 7-18.

11. Alharbi A, Alqwaiee M, Al-Hindi M, Mosalli R, Al-Shamrani A, Alharbi S, et al. Bronchiolitis in children: The Saudi-initiative of bronchiolitis diagnosis, management, and prevention (SIBRO). Ann Thorac Med 2018; 13: 127-143.

12. Del Vecchio A, Franco C, Del Vecchio K, Umbaldo A, Capasso L, Raimondi F. RSV prophylaxis in premature infants. Minerva Pediatr 2018; 70: 579-588.

13. Acero-Bedoya S, Wozniak PS, Sánchez PJ, Ramilo O, Mejias A. Recent Trends in RSV immunoprophylaxis: clinical implications for the infant. Am J Perinatol 2019; 36: S63-S67.

14. Luna MS, Manzoni P, Paes B, Baraldi E, Cossey V, Kugelman A, et al. Expert consensus on palivizumab use for respiratory syncytial virus in developed countries. Paediatr Respir Rev 2020; 33: 35-44.

15. American Academy of Pediatrics Committee on Infectious Diseases, American Academy of Pediatrics Bronchiolitis Guidelines Committee. Updated guidance for palivizumab prophylaxis among infants and young children at increased risk of hospitalization for respiratory syncytial virus infection. Pediatrics 2014; 134: e620-e638.

16. American Academy of Pediatrics. Red book. In: Pickering LK, Baker CJ, Kimberlin DW, Long SS, editors. 2009 Report of the Committee on Infectious Diseases. 28th ed. Elk Grove Village (IL): American Academy of Pediatrics; 2009.

17. Fagbo SF, Garbati MA, Hasan R, AlShahrani D, Al-Shehri M, AlFawaz T, et al. Acute viral respiratory infections among children in MERS-endemic Riyadh, Saudi Arabia, 2012-2013. J Med Virol 2017; 89: 195-201.

18. Jamjoom GA, Al-Semrani AM, Board A, Al-Frayh AR, Artz F, Al-Mobaireek KF. Respiratory syncytial virus infection in young children hospitalized with respiratory illness in Riyadh. $J$ Trop Pediatr 1993; 39: 346-3469.

19. Al-Hajjar S, Akhter J, Al Jumaah S, Qadri SMH. Respiratory viruses in children attending a major referral centre in Saudi Arabia. Ann Trop Paediatr 1998; 18: 87-92.

20. Meqdam MM, Subaih SH. Rapid detection and clinical features of infants and young children with acute lower respiratory tract infection due to respiratory syncytial virus. FEMS Immunol Med Microbiol 2006; 47: 129-133.

21. Dhama K, Khan S, Tiwari R, Sircar S, Bhat S, Malik YS, et al. Coronavirus disease 2019-COVID-19. Clin Microbiol Rev 2020; 33: 1-48.

22. John Hopkins University \& Medicine. COVID-19 Dashboard by the Center for Systems Science and Engineering (CSSE). (Updated 2021; Cited). Available from URL: https://systems. jhu.edu

23. Xiang B, Li P, Yang X, Zhong S, Manyande A, Feng M. The impact of novel coronavirus SARS-CoV-2 among healthcare workers in hospitals: An aerial overview. Am J Infect Control 2020; 48: 915-917.

24. Lambert PH, Ambrosino DM, Andersen SR, Baric RS, Black $\mathrm{SB}$, Chen RT, et al. Consensus summary report for CEPI/BC March 12-13, 2020 meeting: Assessment of risk of disease enhancement with COVID-19 vaccines. Vaccine 2020; 38: 4783-8791. 
25. Yezli S, Khan A. COVID-19 social distancing in the Kingdom of Saudi Arabia: Bold measures in the face of political, economic, social and religious challenges. Travel Med Infect Dis 2020; 37: 101692.

26. Alrashed S, Min-Allah N, Saxena A, Ali I, Mehmood R. Impact of lockdowns on the spread of COVID-19 in Saudi Arabia. Informatics Med Unlocked 2020; 20: 100420.

27. Alsofayan YM, Althunayyan SM, Khan AA, Hakawi AM, Assiri AM. Clinical characteristics of COVID-19 in Saudi Arabia: A national retrospective study. J Infect Public Health 2020; 13: 920-925.

28. Alyami MH, Naser AY, Orabi MAA, Alwafi H, Alyami HS. Epidemiology of COVID-19 in the Kingdom of Saudi Arabia: An Ecological Study. Front Public Heal 2020; 8: 506.

29. Nezar Kobeisy SA, Harbi S Al, Mehdawi RS, Bashammakh DS. Pediatric COVID-19 Patients in Jeddah, Saudi Arabia: Clinical, Laboratory and Radiological Aspects. J Biomed Sci 2020; 9: $1-4$.

30. Heald-Sargent T, Muller WJ, Zheng X, Rippe J, Patel AB, Kociolek LK. Age-related differences in nasopharyngeal severe acute respiratory syndrome coronavirus 2 (sars-cov-2) levels in patients with mild to moderate coronavirus disease 2019 (COVID-19). JAMA Pediatr 2020; 174: 902.
31. Ministry of Health. Neonatology Services Improvement Program. Guidelines for neonates born to mothers with suspected or confirmed COVID-19 infection. (Updated 2021. Cited). Available from URL: https://www.moh.gov.sa/Ministry/ MediaCenter/Publications/Documents/Saudi-Guideline-forNeonate.pdf

32. Alrabiaah A, Alshaer A, Estrella SM, Inclan KA, Aljammaz H, Almoosa K, et al. Effects of the Coronavirus disease 2019 pandemic on routine pediatric immunization coverage rates at the main University Hospital in Saudi Arabia. Saudi Med J 2020; 41: 1197-1203.

33. AstraZeneca. US FDA grants Breakthrough Therapy Designation for potential next-generation RSV medicine MEDI8897. [Updated 2021; Accessed 2021 February 10]. Available from URL: https://www.astrazeneca.com/media-centre/pressreleases/2019/us-fda-grants-breakthrough-therapy-designationfor-potential-next-generation-rsv-medicine-medi8897.html\#

34. Griffin MP, Yuan Y, Takas T, Domachowske JB, Madhi SA, Manzoni P, et al. Single-dose nirsevimab for prevention of RSV in preterm infants. N Engl J Med 2020; 383: 415-425. 